\title{
The sky as a laboratory: an educational project of the Department of Astronomy of the University of Padova
}

\author{
S. Ciroi, F. Di Mille and P. Rafanelli \\ Department of Astronomy, Padova University, \\ Vicolo dell'Osservatorio 3, 35122 Padova, Italy \\ email: stefano.ciroi@unipd.it \\ email: francesco.dimille@unipd.it \\ email: piero.rafanelli@unipd.it
}

\begin{abstract}
The Sky as a Laboratory" is an educational project of the Department of Astronomy of the University of Padova aimed to give students a physical approach to astronomy and astrophysics. It is a regional program designed to improve science education in the last two grades of high school, by creating cooperation between scientists and teachers. Currently it is present in 30 high schools around all provinces of the region of Veneto in the North-East of Italy. The close involvement in the didactical activities of high schools teachers is without any doubt the winning strategy of the project. Their enthusiastic participation to teaching and organising activities attracts each year the attention for sciences of an increasing number of students and suggests new ideas for future educational activities.
\end{abstract}

Keywords. Education, Astronomy, High Schools

\section{Introduction}

When coming to university many Italian students have the feeling that astronomy is essentially a qualitative and descriptive science. Their disappointment is bitter when they discover that astronomy is in fact mathematics and physics applied to the study of celestial phenomena. This is due essentially to the following reasons: 1) astronomy is limited to high schools focusing on sciences and/or humanities; 2) it is generally limited to the last year, when students are 18-19 years old; 3) it is not directly connected to Physics and Mathematics.

On the basis of this experience we decided to develop an educational project with completely new characteristics with respect to the many other Italian outreach activities dedicated to students of different levels. This project named The Sky as a Laboratory was designed to improve science education in the last two grades of high school, by creating cooperation between scientists and teachers. It offers the students: 1) a direct and personal experience of astronomical observations at the telescope followed by quantitative data analysis; 2) a learning environment where they act in first person; 3) a general practical knowledge of the use of instruments like spectrographs, imaging cameras, CCDs, as well as dedicated astronomical software and new operating systems; 4) a practice with multiple tools for producing papers and presentations on the scientific results obtained.

\section{The Sky as a Laboratory}

The Sky as a Laboratory has three main goals: 
(a) to give the students a physical approach to astronomy and astrophysics;

(b) to point out the interdisciplinary nature of astronomy and astrophysics, stressing that many of the open questions on the interpretation of celestial phenomena are connected with other sciences, such as chemistry, geology and biology;

(c) to offer guidelines to the students for the choice of their future field of study at the university, giving them the possibility to raise awarness on their attitude (or not) for science.

The Sky as a Laboratory started in 2001 as a pilot project. At the beginning it was decided to involve only two schools in order to test the effective interest of the students. In 2002 we organized a structured project: we first contacted teachers working in high schools of the Veneto region having a degree or even a Ph.D. in astronomy and then we started with them a close collaboration. Their institutes became official reference schools for the project, that is, the schools where advanced lectures of astrophysics were offered to the students. We also carried out for the first time lab activities at the Asiago Astrophysical Observatory (Fig. 1) with the students attending the project. The project was successful and since 2003 it was extended to all the schools of the Veneto region which were interested. Through this action the number of associated schools, involved teachers and participating students rapidly increased, but it was also necessary to dedicate a long time to newly define the full structure of the project and improve the quality of the activities in Asiago.

Every year around 350-400 students from 30 high schools in the Veneto region freely decide to take part in the project and attend an advanced course consisting of 14 hours of astrophysical lectures and a conference, which are given by the staff of the Dept. of Astronomy in Padova and the involved teachers in the 9 reference schools. More than 30 teachers and several principals are involved in the project. Around 150-170 students per year decide to attempt the admission test after having attended the astrophysical lectures. Fifty of them per year are admitted to the training period at the Asiago Astrophysical Observatory. The duration of the training ranges between 35 and 40 hours required for observations, data reduction, data analysis and interpretation. Finally 12-13 scientific reports per year are produced by the students, focused on several astrophysical topics.
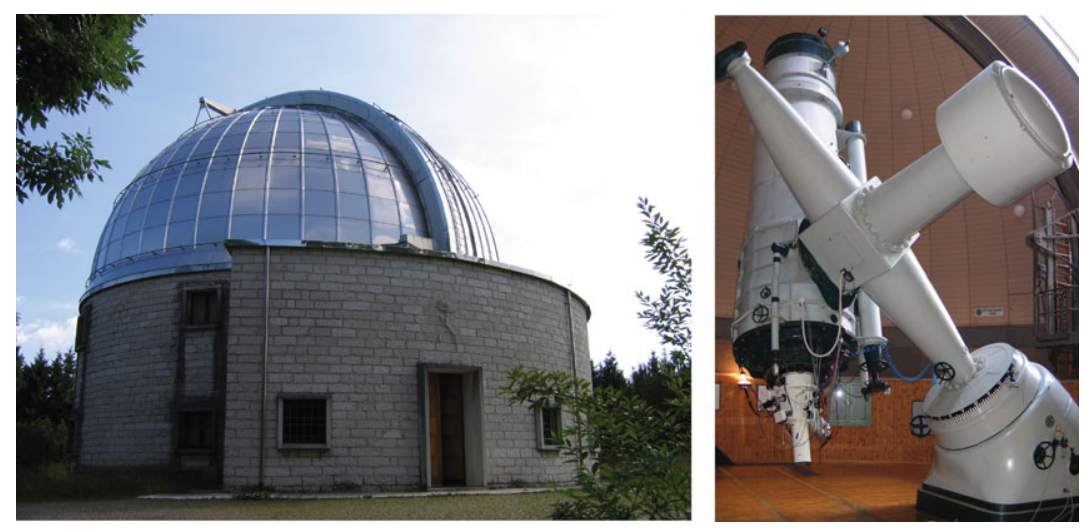

Figure 1. Dome and telescope of the Asiago Astrophysical Observatory. 


\section{The project in detail}

The Sky as a Laboratory officially starts in October. During the first two months the students who have registered attend a course of 7 lectures (14 hours) of basics of astronomy and astrophysics (1 lecture per week). Currently the topics of the lectures are:

(a) The blackbody radiation

(b) The dual nature of light and the hydrogen atom

(c) Magnitudes, colours and spectra of stars

(d) The H-R diagram and stellar evolution

(e) The planets of the Solar System and the search of extrasolar planets

(f) Gaseous nebulae spectra

(g) The universe of galaxies

In addition the project envisages a conference by a scientist of the Dept. of Astronomy, often on topics different from the lectures and closer to modern reaserch.

In December, a public conference for all participating students and teachers is organised at the Great Hall of the University of Padova. Internationally renowned astronomers are invited to give a talk.

In January a test with 42 multiple-answer questions is scheduled for the admission to a training period at the Asiago Astrophysical Observatory. Every year about 150-170 students decide to attempt this test, but only a third of them, 50-60 students, is selected for the second phase of the project.

The month of the training period in Asiago is February. Four groups of 13-15 students are organised and each group spends 3 days at the Observatory, including nights of direct observations at the Galileo $1.2 \mathrm{~m}$ telescope equipped with a professional spectrograph and modern CCD detectors.

The students must write conclusive reports in scientific article format, which are then collected in a volume edited every year by the Dept. of Astronomy. In April they show their works to their colleagues teachers as powerpoint presentations at the Seminar Room of the Dept. during the final day of the project.

\section{Training at the telescope}

The Asiago Astrophysical Observatory facility consists of the old $1.2 \mathrm{~m}$ telescope (D $=122 \mathrm{~cm}, \mathrm{~F}=1950 \mathrm{~cm}$ ), completely refurbished, equipped with a Boller \& Chivens Spectrograph. Four gratings are available, having 150, 300, 600 and 1200 lines/mm, which produce spectra with a resolution of $16,8,4$ and $2 \AA$, respectively (using a slit width of $\left.2^{\prime \prime}\right)$. A modern and professional CCD detector Andor iDUS $512 \times 2048$ pixels $(13.5 \mu / \mathrm{px})$ allows to obtain high quality spectra of relatively faint sources: magnitude $15-16$ can be reached in $0.5-1 \mathrm{hr}$ of total exposure time. The spatial scale on the CCD is 0 .' 6 / pixel.

Both telescope and spectrograph are operated from a control room located at the ground floor of the dome. This room also contains computers with Windows/Linux operating systems, a printer and a beamer useful both for simple lectures and for guiding the students during their activities. Computers are equipped with professional astronomical software, like IRAF, MIDAS, DS9, SMongo, Sextractor, Galfit and Topcat.

Up to 15 students at a time can be hosted for lab activities. First, the students are grouped on the basis of different topics of research proposed by the astronomers during a briefing with them and their teachers. Then, the observation at the telescope can start. 
During the night, if weather conditions are acceptable, students can directly observe, pointing the telescope and acquiring the data necessary for their research. Otherwise, datasets already prepared for reduction, when necessary, and analysis are extracted either from the local archive or from public archives (like SDSS, HST, DSS, 2MASS, etc.). During daytime the students carry out data reduction under the guidance of the astronomers, who explain the procedure step by step. Data analysis is performed using astronomical software and taking advantage of scientific information extracted from NASA/ADS Abstract Service and internet web sites.

Here is a list a research topics already tested and developed by using both observed and archival data:

\section{- SOLAR SYSTEM}

- Spectral analysis of absorption features of planets and satellites

- Rotation curve and mass determination of planets

- Spectral analysis of comets

- STARS

- H-R and colour-colour diagrams of open and globular clusters

- Light curves of variable stars

- Spectral classification of main sequence stars

- Temperature determination of stars

- Supernovae classification

- NEBULAE

- Temperature, density and chemical composition of Galactic gaseous nebulae

- Star formation rate in extragalactic HII regions

- GALAXIES

- Morphology of nearby galaxies

- H-R diagrams of dwarf galaxies of the Local Group

- Stellar populations in galaxies

- Mass estimate of spiral and elliptical nearby galaxies

- Spectroscopy of active nuclei and starburst galaxies

- Photometric redshifts

- Optical counterparts of X-ray sources

- Star formation in spiral galaxies

As an example, the recent work by Monai et al. (2009) was carried out with archival data extracted from the Sloan Digital Sky Survey Data Release 6 http://www.sdss.org/dr6: Relations between stellar temperature, color indices and equivalent widths of spectral lines.

The students have firstly selected a sample of 26 stars having different color index $g-r$, then they have downloaded the spectra of such stars and have fitted by eye the continuum of each stellar spectrum by applying the Planck's equation through a script prepared by the astronomers for SMongo environment (Fig. 2). They plotted the inverse of the so-estimated temperatures againts the corresponding color indices with TOPCAT and performed a linear regression obtaining a strong correlation (Fig. 3). With this formula, they could verify that the color index of a star is directly related to its temperature, but also they could extract photometric information of 100000 stars and after having converted $g-r$ values into temperatures they plotted a histogram of temperatures useful to give a statistical distribution of the stellar spectral types (Fig. 4). 


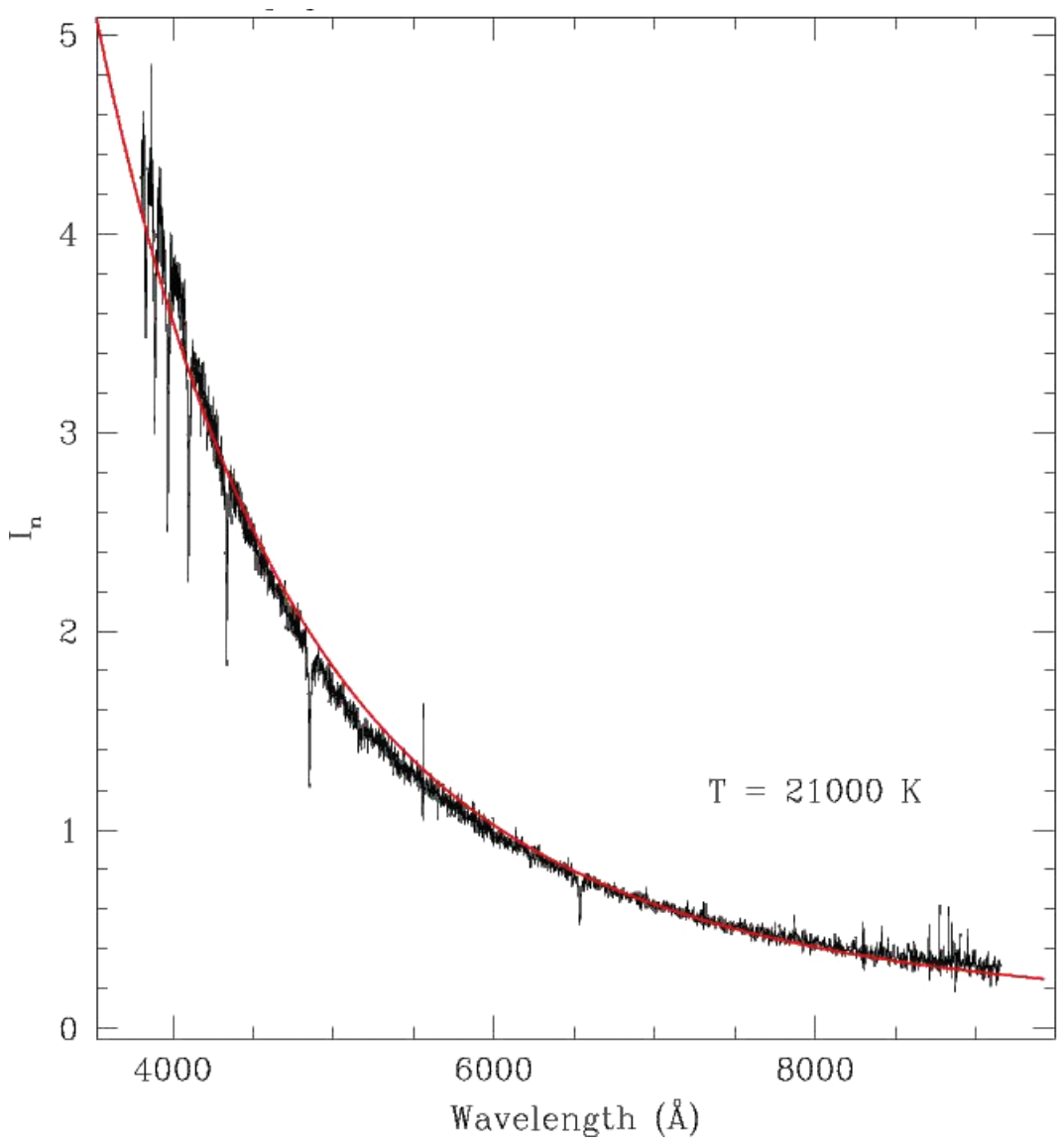

Figure 2. Spectrum of a hot star. The red line is the model based on Planck's equation.

\section{Products of the project}

So far The Sky as a Laboratory has produced the following material:

- A website (English version is under way) collecting all information about the project and in particular the material useful for lectures and available to everybody http://www.astro.unipd.it/progettoeducativo.

- Internal publications of the works done by the students during their stay in Asiago, which can easily become examples of possible exercises.

- Scientific publications, such as Ciroi et al. (2009a,b)

- A book of astronomy for students of the high schools and for everybody interested in this topic, written by the teachers with a degree in astronomy and edited by the staff of the project (in press).

- For the first time in Italy, teachers of a high school taking part in the project have been able to start lectures and practice of astronomy since the 1st year (when students are 14-15 years old).

\section{Summary}

[...] In laboratory work: students can use real-life data; they can use the same computer languages and software packages that reaserchers use. The labs should be 


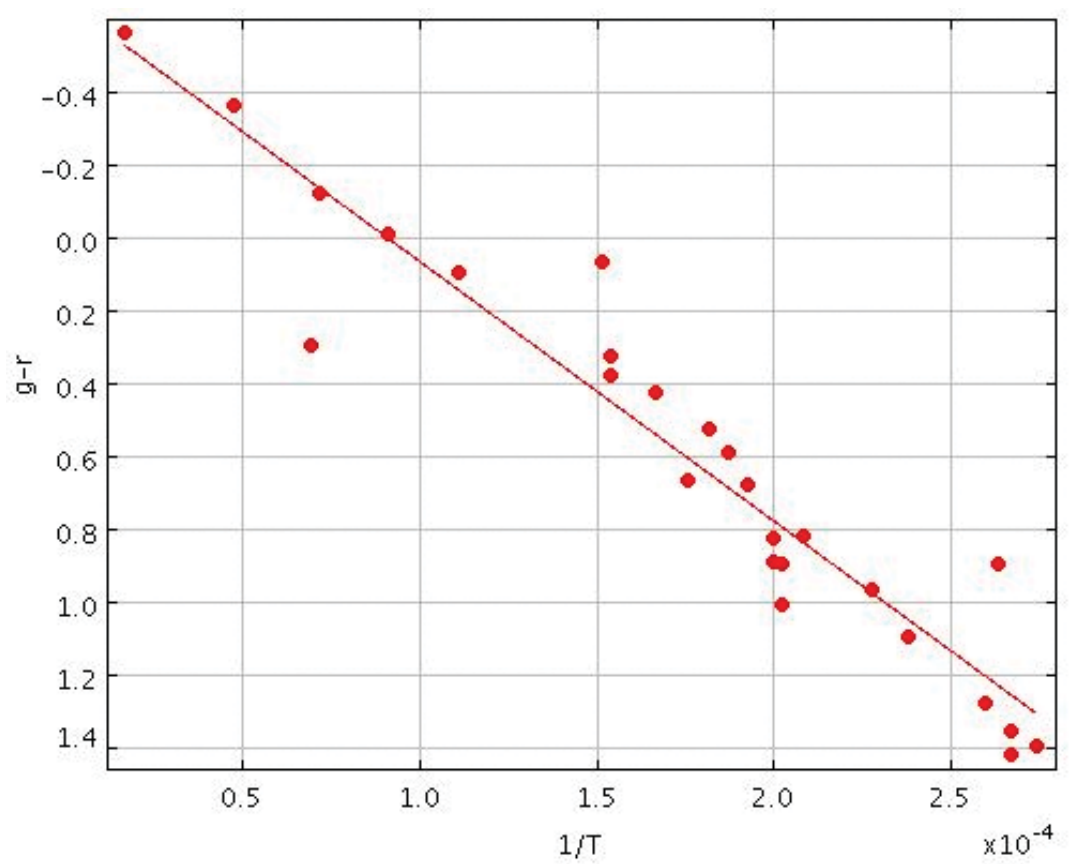

Figure 3. Diagram of inverse temperature against colour. The solid line is the result of the linear regression.

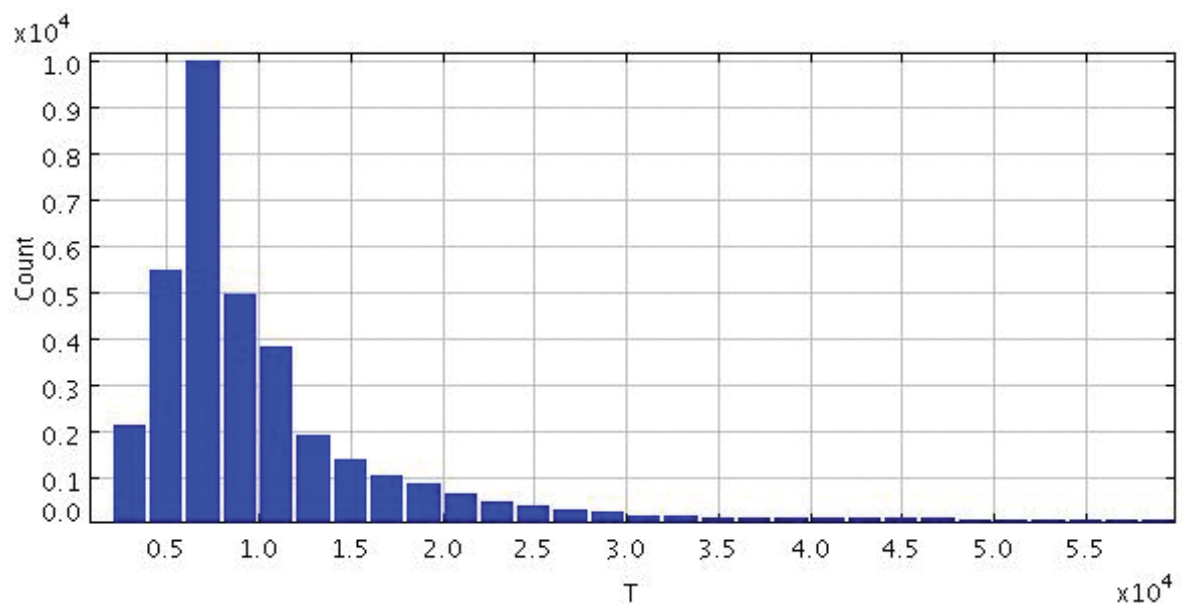

Figure 4. Histogram of temperatures of 100000 stars.

authentic mirroring real research, rather than aiming for a pre-determined cookbook result ... They can learn that the astronomical knowledge is not produced by textbooks but by astronomers, perhaps even by themselves! (Percy 2006) .

The Sky as a Laboratory has demonstrated that it is possible to spread even complicated astrophysical concepts among strongly motivated students of the high school having a basic knowledge of physics and mathematics. The success of this project depends definetely on the enthusiasm and the efforts of the teachers of the involved schools, and on the close collaboration between them and the staff of the Dept. of Astronomy in Padova. 
Students who attended the full project, made an important experience for them, for their life and their future, whatever their future choices will be.

\section{References}

Ciroi, S., Di Mille, F., Zaccaria, M., Vicariotto, L., Pellegrini, C., Zorzan, M., \& Pegoraro, A. 2009, The Astronomer's Telegram, 1985, 1

Ciroi, S., di Mille, F., Carcò, M., Rossi, M., Rosson, P., \& Benetti, S. 2009, Central Bureau Electronic Telegrams, 1697, 2

Monai, E., Pagotto, I., Artusi, E., De Luca, L. 2009, The Sky as a Laboratory, Internal Reports 2009, http://www.astro.unipd.it/progettoeducativo/tesine.html

Percy, J.R. 2006, Innovation in Teaching/Learning Astronomy Methods, 26th meeting of the IAU, Special Session 2, p. 2 